\title{
The Prevalence and Pattern of Abnormal Hysteroscopy Findings among Subfertile Patients at Albayda Fertility Centre/Libya
}

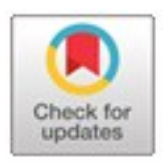

Agzail Elhddad ${ }^{1 *}$ and Zamzam Shaban ${ }^{2}$

$I^{\prime *}$ Albayda Fertility Teaching Centre; Department. of Obstetrics \& Gynecology, Faculty of Medicine,

Omar Al-Mukhtar University, Albayda/ Libya.

${ }^{2}$ Albayda Fertility Teaching Centre, Libya.

Received: 20 February 2020 / Accepted: 06 June 2020

Doi: https://doi.org/10.54172/mjsc.v35i1.212

\begin{abstract}
To estimate the prevalence and types of intrauterine abnormalities in subfertile women: a prospective study was carried out at Albayda Fertility Teaching Centre-Libya. The study was conducted on 115 infertile women attending Albayda Fertility Centre between January and May 2019. Diagnostic hysteroscopy was conducted after initial basic infertility assessment workup to assess the presence and types of uterine cavity pathologies. The duration of infertility ranged from 1-17 years, and the majority of the patients were presented with primary infertility $(62.6 \%), 64.3 \%$ of the women had abnormal findings, and the most common lesion detected was endometrial polyp representing $44.6 \%$ of the lesions, followed by endometritis $17.6 \%$. Seven cases had septum, and 20 patients had more than one pathology. Corrective measures were taken accordingly. $6.1 \%$ of the patients had a spontaneous pregnancy within three months of follow up. The prevalence of abnormal hysteroscopy findings among the studied population was high. Intrauterine endometrial polyps, endometritis, and uterine septum were the most frequent abnormality detected. These findings may indicate a need to incorporate hysteroscopy in the routine evaluation of infertility.
\end{abstract}

Keywords: Hysteroscopy; Infertility; Intrauterine Lesion.

\section{INTRODUCTION}

There is increasing evidence that had emphasized the importance of the uterus and the intrauterine abnormalities on the reproductive outcome (Galliano et al., 2014; Taylor \& Gomel, 2008; Yanaihara et al., 2008). Intrauterine pathologies are found to affect $25 \%$ to $50 \%$ of subfertile patients (El-Mazny et al., 2011; Pundir \& Toukhy, 2010; Taylor \& Gomel, 2008). Therefore, the evaluation of the uterine factor is an important step to be taken in the assessment of infertility (Cicinelli et al., 2015; Polisseni et al., 2003; Taylor \& Gomel, 2008). Hysteroscopy, saline infusion sonography (SIS), transvaginal sonography (TVS), and hysterosalpingography (HSG), have been used to assess the uterine cavity. Hysteroscopy offers the op- portunity for the direct visualization of the uterine cavity under magnification. It also allows the surgical correction of the identified pathology (Zinna et al., 2015). Performing a diagnostic hysteroscopy and correction of defined pathology were found to improve significantly the pregnancy rate in patients with previously failed in-vitro fertilization (IVF) trails (Demirol \& Gurgan, 2004; Raju et al., 2006).

Moreover, saline irrigation and mechanical manipulation of the endometrium may enhance the endometrial receptivity (Almog et al., 2010; Potdar et al., 2012), and just the passage of the tip of the hysteroscopy through the cervical os makes the embryo transfer easier during IVF trails, suggesting that just performing the procedure has a pos-

\footnotetext{
*Corresponding Author: Agzail Elhddad Agzail.elhddad@gmail.com, Albayda Fertility Teaching Centre; Department. of Obstetrics \& Gynecology,
} Faculty of Medicine, Omar Al-Mukhtar University, Albayda/ Libya. 
itive prognostic effect on fertility.

In the developing countries, reports on the use of hysteroscopy in the management of infertility in public hospitals are scarce (Ugboaja et al., 2018) and the case is the same in Libya. In addition, hysteroscopy has commenced recently in our center and no previous study has been done yet on the role of hysteroscopy in infertility management. Therefore, this study was conducted to evaluate the prevalence and type of intrauterine lesions at hysteroscopy among infertile women in our setting.

\section{MATERIALS AND METHODS}

A prospective study was conducted at Albayda Fertility Centre - Public Teaching Centre in Libya (afc.med.ly) in the period from January 2019 to May 2019 after obtaining the ethical approval from the local ethics committee and written consent was signed by the couples before the commencement of the procedure. Diagnostic hysteroscopy was conducted after initial basic infertility assessment workup for all included couples.

Participants were infertile women, with or without uterine cavity pathologies suspected or diagnosed by transvaginal sonography, hysterosalpingography, saline infusion sonography, or previous hysteroscopy. The included women were either enrolled during the basic infertility investigation and before being scheduled for any Assistant Reproductive Technology (ART) or undergone one or more failed ART trails. Women with ongoing or recent pelvic infection were not included in the study.

Hysteroscopy (STORZ Co.) was performed in Operation Theatre under anesthesia in the immediate postmenstrual phase (day 6 to 11 of the menstrual cycle) for better visualization of the uterine cavity. A flexible, singleflow hysteronvideoscopy with an outer diameter of $6 \mathrm{~mm}$ was introduced transcervi- cally. Normal saline delivered by a drip suspended one meter above the patient's head was used as distending media. The endocervical canal was inspected at the beginning of the procedure. Once inside the uterine cavity, a systematic examination was performed by a general evaluation of the uterine cavity starting from the isthmic region. A detailed and systematic examination done for the uterine cavity, the uterine cornua, tubal ostia, uterine fundus, anterior, posterior, and lateral uterine walls and also the endocervical canal was evaluated. The criteria taken for normal hysteroscopy were; normal uterine cavity (normal shape and contour, with no local pathology such as myoma or polyp), normal endometrium, and visible bilateral ostia. The visualizable "flow" effect in the uterine tube ostia during hysteroscopic examination has been found to be predictive of tubal patency (Promberger et al., 2018).

Endometrial scratch was done for almost all cases, and an endometrial biopsy was taken when indicted and sent for histopathological evaluation. Abnormalities of the uterine cavity, endometrium, and uterine ostia were noted and recorded on a special data form; corrective measures were taken accordingly using either scissors, forceps or both. At the end of the procedure, TVS was done, and the visualization of free fluid in the pelvis suggests the patency of at least one Fallopian tube. Prophylactic antibiotics were given to the patients before the start of the procedure. The patients were kept under observation for a minimum of two hours for a full recovery and to assess any possible side effects and complications.

The primary outcome measures were the type and the frequency of abnormal findings during the hysteroscopy. The secondary outcomes were, any correction done during the procedure, any complications aroused during or after the procedure as well as the reproductive outcome. 
Statistical Analysis: SPSS package version 25 (SPSS Inc., Chicago, IL, USA) was used for data entry and statistical analysis. Shapiro-Wilk test was used to assess the normality of data. The mean (SD) and median were calculated for the continuous variables according to data distribution, while percentages were calculated for the composite variables. The continuous variables were compared using the independent $t$-test. The categorical variables were compared for significant differences using the Chi-square test, were a $P$-value of $<0.05$ was considered to be significant. The results were presented in tables, figures or both.

\section{RESULTS}

The indication for hysteroscopy was the evaluation of the uterine cavity in 115 infertile women regardless of previous ultrasound scan or HSG findings. Figure 1 demonstrates the etiology of infertility in the study population. The included females were aged between 18 and 45 years. The (median: 34) with a mean body mass index (BMI) of 28.8 . The duration of infertility in the patient cohort ranged from 1 to 17 years (median, 5 years). The majority were presented with primary infertility $62.6 \%(n=72)$. Out of these 115 patients; 8 were having a recurrent miscarriage, and 47 were with previously failed ART trails and one patient with repeated preterm labor.

Table 1 summarized the previous infertility treatment received and history of previous pelvic and abdominal surgery; $91.4 \%$ of the included women received various modalities of infertility treatment. 55 patients had no history of pelvic or lower abdominal surgery. Appendectomy and caesarian sections were the most common surgeries done in the remaining 60 women.

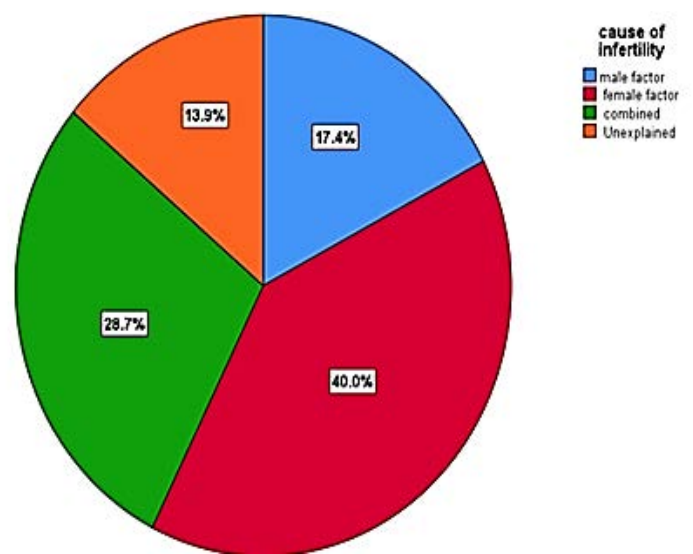

Figure: (1). Causes of infertility

Table: (1). Past infertility and surgical treatment

\begin{tabular}{ll}
\hline \hline & No. (\%) \\
\hline PSH & No $55(47.8 \%)$ \\
& Yes $60(52.2 \%)$ \\
Type of PSH: & \\
Cesarean section & $14(28 \%)$ \\
Myoma & $3(6 \%)$ \\
Ovarian cyst & $5(10 \%)$ \\
Lower abdominal surgery & $28(56 \%)$ \\
Infertility treatment & No $9(8.5 \%)$ \\
& Yes $96(91.4 \%)$ \\
Type of infertility treatment: & \\
Ovulation induction & $51(53 \%)$ \\
IUI & $21(22 \%)$ \\
ICSI & $19(19.7 \%)$ \\
IUI+ICSI & $5(5.2 \%)$ \\
\hline \hline PSH: past pelvic and lower abdominal surgery \\
IUI: intrauterine insemination ICSI: intacytoplasmic sperm injec- \\
tion
\end{tabular}

Table 2 shows that; out of all the hysteroscopies, no intrauterine pathology was detected in 41 women $(35.7 \%$ of the study population). $74 / 115$ (64.3\% of the participants) had abnormal findings. The most commonly detected pathology was single or multiple endometrial polyps, identified in 33 cases $(44.6 \%)$. The second most common lesion was endometritis $(17.6 \% ; n=13 / 74)$. The presence of endometritis was based on the hysteroscopic visualization of stromal edema, endometrial hyperemia, or micropolyps of less than $1 \mathrm{~mm}$ (Cicinelli et al., 2005) and the presence of plasma cells in the endometrial stroma (Greenwood \& Moran, 1981). Both pathologies (endometrial polyp 
and endometritis) were found in $16.2 \%$ of cases. 7 cases were diagnosed with a septum (6\%), and 8 cases were found to have a septum with a polyp or with endometritis. Only one case with intrauterine adhesions (IUAs) was detected. Other findings were; 18 cases with unilateral or bilateral occluded ostia, and 7 cases with cervical synechia. No cases with submucous myomas were detected.

Table: (2) hysteroscopic findings

\begin{tabular}{ll}
\hline \hline Hysteroscopic finding & No. $(\%)$ \\
\hline Normal & $41(35.7 \%)$ \\
Intrauterine pathology:- & $74(64.3 \%)$ \\
Polyp (single/multiple) & $33(44.6)$ \\
Endometritis & $13(17.6 \%)$ \\
Endometritis+ polyp & $12(16.2 \%)$ \\
Septum & $7(6 \%)$ \\
Septum+ endometritis & $5(4.3 \%)$ \\
Septum+ polyp & $3(2.6 \%)$ \\
\hline \hline
\end{tabular}

There was no significant difference between those with and without intrauterine pathology detected during hysteroscopy in a past pelvic and lower abdominal surgery. However, those with pathology were having a significantly higher rate of miscarriage and recurrent miscarriage as demonstrated in $\mathrm{Ta}$ ble 3 .

Table (3): past surgical and obstetric characteristics of the included infertile patients

\begin{tabular}{llll}
\hline \hline & No pathology & With pathology & Chi-square \\
\hline PSH & $17 / 41(41 \%)$ & $38 / 68(56 \%)$ & 0.23 \\
Miscarriage & $2 / 41(4.9 \%)$ & $23 / 74(31.1 \%)$ & $<0.001$ \\
Recurrent mis & 00 & $8 / 72(10.8 \%)$ & $<0.001$ \\
riage & & & \\
\hline \hline
\end{tabular}

PSH: past pelvic and lower abdominal surgery

Surgical treatment of the lesions was carried out accordingly at the same sitting as shown in Table 4; polypectomy $[\mathrm{n}=35 ;(47.3 \%)]$, metroplasty $[\mathrm{n}=11 ;(14.9 \%)]$. Proximal tubal cannulation and release of the cervical strictures ( $n=12$ and 7 cases respectively), and in eight cases more than one procedure was done.
Table (4): surgical procedures done during the hysteroscopy

\begin{tabular}{ll}
\hline \hline Procedure & No. $\%$ \\
\hline Nothing & $41(35.6 \%)$ \\
Polypectomy & $35(47.3 \%)$ \\
Metroplasty & $11(14.9 \%)$ \\
Adhesiolysis & $1(1.4 \%)$ \\
Proximal ostia canalization & $12(16.2 \%)$ \\
More than one procedure & $8(10.8 \%)$ \\
Release of cervical stricture & $7(9.5 \%)$ \\
\hline \hline
\end{tabular}

No complication such as uterine perforation, hemorrhage, or postoperative fever was recorded. Within three months of follow up, seven out of 115 cases $(6.1 \%)$ of the patients had a spontaneous pregnancy after the procedure; four cases after polypectomy, two cases after metroplasty, and one case after proximal canalization of the ostia.

\section{DISCUSSION}

Uterine congenital anomalies, as well as acquired diseases, can adversely impact endometrial receptivity, which results in implantation failure and may manifest as infertility or bad obstetric outcome. Hysteroscopy was found to be superior to HSG and transvaginal scan in the detection of unsuspected uterine pathologies (Elsetohy et al., 2015). Moreover, the pregnancy rate was improved upon the correction of the detected pathology (Alleyassin et al., 2017; Makrakis \& Pantos, 2010; Shohayeb \& El-Khayat, 2012). Therefore, many assistance reproductive centers have incorporated the uterine cavity evaluation in their basic fertility workup because of the high incidence of unsuspected pathologies (Elsetohy et al., 2015). However, international institutions, such as NICE guidelines on fertility assessment and treatment, have not included the uterine cavity assessment in their guidelines unless clinically indicated, because the treatment of these abnormalities has not been justified on improving reproductive outcome (for Women's \& Children's Health, 2013).

The rate of the intrauterine lesions detected 
by hysteroscopy in women with no abnormalities detected by ultrasound scan reported to be $10.9 \%$ (Fatemi et al., 2010) and $22.9 \%$ (Karayalcin et al., 2010) and up to $41.1 \%$ (Elbareg \& Essadi, 2017). In the current study, the detection rate was $64.3 \%$ (74/115). This high rate could be due to the inclusion of all patients with or without previously diagnosed or suspected pathologies by ultrasound scan, HSG, or both. The prevalence of lesions detected in the present study was lower than the prevalence of $70.4 \%$ reported by a study conducted in $\mathrm{Ni}$ geria (Ugboaja et al., 2019), and 69.7\% prevalence reported by another study conducted in Egypt in the same year (Farag et al., 2019). This high prevalence of intrauterine pathologies in the study conducted in Nigeria may be explained by a high incidence of intrauterine adhesions (47.8\%) that could be a consequence of post-traumatic or post-infectious damage to the endometrial basal layer resulting in endometrial fibrosis and uterine cavity obliteration. However, endometritis was the least reported pathology in the study done in Egypt.

Not only the prevalence of intrauterine lesions detected by hysteroscopy was different between previous studies, but also the frequency of different detected abnormalities. Findings on hysteroscopy among the studied women comprised mainly of endometrial polyps, endometritis, and uterine septum. Endometrial polyps were the most common pathology; identified in 33/74 (44.6\%), followed by endometritis in 13/74 (17.6\%), and 12 patients had both endometrial polyps and endometritis. 7/74 women were diagnosed with septa. Also, 8/74 had septa with other pathologies. Only one case was diagnosed with intrauterine adhesions and there was no case of myoma.

The endometrial polyp was the commonest pathology in another study (Yang et al., 2019), and myoma was the least reported le- sion. The intrauterine adhesions were the commonest pathology, followed by polyps, then myomas, and lastly, Mullerian tube defect during the diagnostic hysteroscopy, as reported by another two studies (Elbareg \& Essadi, 2017; Ugboaja et al., 2019).

The high prevalence rate of intrauterine lesions diagnosed and treated during hysteroscopy and the spontaneous pregnancy of $6.1 \%$ during three months of follow up in the current study could support the hypotheses that intracavitary pathologies, i.e., endometrial polyps (Galliano et al., 2014; Pundir \& Toukhy, 2010) and submucous fibroids (Bosteels et al., 2015; Galliano et al., 2014; Pritts, 2001), play an important role in females with previous reproductive failure. Therefore, identifying and correcting intrauterine lesions during hysteroscopy could improve reproduction outcomes.

Hysteroscopy remains a relatively safe procedure, and no complications were recorded during or after the procedure in the present study. Contrarily, complications were reported by others (Bradley, 2002; Pasini \& Belloni, 2001). However, these studies were performing surgical hysteroscopy, and as mentioned before, diagnostic hysteroscopy has fewer risks than surgery (Bradley, 2002). Nevertheless, the complications in operative hysteroscopy are infrequent and are usually easy to manage.

The high detection rate of intrauterine abnormalities and the safety of the procedure suggest a need to incorporate diagnostic hysteroscopy in the routine assessment of female infertility, as this may improve the infertility management outcome.

Future work to investigate the accuracy of hysteroscopy in comparison to highresolution $2 \mathrm{D}$ ultrasound scan and hysterosalpingography in the detection of intrauterine pathologies will be conducted. 


\section{CONCLUSION}

The prevalence of abnormal hysteroscopy findings among the studied population was high. Endometrial polyps, endometritis, and uterine septum were the most frequently detected abnormality. These findings may indicate a need to incorporate hysteroscopy in the routine evaluation of infertility, as this may improve the infertility management outcome.

\section{REFERENCES}

Alleyassin, A., Abiri, A., Agha-Hosseini, M., \& Sarvi, F. (2017). The value of routine hysteroscopy before the first intracytoplasmic sperm injection treatment cycle. Gynecologic and obstetric investigation, 82(2), 125-130 .

Almog, B., Shalom-Paz, E., Dufort, D., \& Tulandi, T. (2010). Promoting implantation by local injury to the endometrium. Fertility and sterility, 94(6), 2026-2029.

Bosteels, J., Kasius, J., Weyers, S., Broekmans, F. J., Mol, B. W. J., \& D'Hooghe, T. M. (2015). Hysteroscopy for treating subfertility associated with suspected major uterine cavity abnormalities. Cochrane Database of Systematic Reviews(2.)

Bradley, L. D. (2002). Complications in hysteroscopy: prevention, treatment and legal risk. Current Opinion in Obstetrics and Gynecology, 14(4), 409-415.

Cicinelli, E., Matteo, M., Tinelli, R., Lepera, A., Alfonso, R., Indraccolo, U., Marrocchella, S., Greco, P., \& Resta, L. (2015). Prevalence of chronic endometritis in repeated unexplained implantation failure and the IVF success rate after antibiotic therapy. Human Reproduction, 30(2), 323-330 .

Cicinelli, E., Resta, L., Nicoletti, R., Tartagni, M., Marinaccio, M., Bulletti, C., \& Colafiglio, G. (2005). Detection of chronic endometritis at fluid hysteroscopy. Journal of minimally invasive gynecology, 12(6), 514-518 .

Demirol, A., \& Gurgan, T. (2004). Effect of treatment of intrauterine pathologies with office hysteroscopy in patients with recurrent IVF failure. Reproductive biomedicine online, $8(5)$, 590-594.

El-Mazny, A., Abou-Salem, N., El-Sherbiny, W., \& Saber, W. (2011). Outpatient hysteroscopy: a routine investigation before assisted reproductive techniques? Fertility and sterility, 95(1), 272-276.

Elbareg, A., \& Essadi, F. M. (2017). Impact of routine hysteroscopy (HS (prior to intrauterine insemination (IUI) on pregnancy rates (PR) among infertile couples at Al-Amal Hospital, Misurata, Libya. J Pregnancy Reprod, 1(4), 1-4 .

Elsetohy, K. A. A. A., Askalany, A. H., Hassan, M., \& Dawood, Z. (2015). Routine office hysteroscopy prior to ICSI vs. ICSI alone in patients with normal transvaginal ultrasound: a randomized controlled trial. Archives of gynecology and obstetrics, 291(1), 193-199.

Farag, A. H., Salama, M. H., \& Badrous, E. S. (2019). Assessment of the prevalence of abnormal hysteroscopic findings in infertile women undergoing ART. The Egyptian Journal of Hospital Medicine, 75(3), 2433-2440 . 
Fatemi, H., Kasius, J., Timmermans, A., Van Disseldorp, J., Fauser, B., Devroey, P., \& Broekmans, F. (2010). Prevalence of unsuspected uterine cavity abnormalities diagnosed by office hysteroscopy prior to in vitro fertilization. Human reproduction, 25(8), 1959-1965 .

for Women's, N. C. C., \& Children's Health, U. (2013). Fertility: assessment and treatment for people with fertility problems .

Galliano, D., Bellver, J., Díaz-García, C., Simón, C., \& Pellicer, A. (2014). ART and uterine pathology: how relevant is the maternal side for implantation? Human reproduction update, 21(1), 13-38 .

Greenwood, S. M., \& Moran, J .J. (1981). Chronic endometritis: morphologic and clinical observations. Obstetrics \& Gynecology, 58(2), 176-184 .

Karayalcin, R., Ozcan, S., Moraloglu, O., Ozyer, S., Mollamahmutoglu, L., \& Batioglu, S. (2010). Results of 2500 office-based diagnostic hysteroscopies before IVF. Reproductive biomedicine online, 20(5), 689-693 .

Makrakis, E., \& Pantos, K. (2010). The outcomes of hysteroscopy in women with implantation failures after in-vitro fertilization: findings and effect on subsequent pregnancy rates .Current Opinion in Obstetrics and Gynecology, 22(4), 339-343.

Pasini, A., \& Belloni, C. (2001). Intraoperative complications of 697 consecutive operative hysteroscopies. Minerva ginecologica, 53(1), 13-20 .
Polisseni, F., Bambirra, E. A., \& Camargos ‘ A. F. (2003). Detection of chronic endometritis by diagnostic hysteroscopy in asymptomatic infertile patients. Gynecologic and obstetric investigation, 55(4), 205-210 .

Potdar, N., Gelbaya, T., \& Nardo, L. G. (2012). Endometrial injury to overcome recurrent embryo implantation failure: a systematic review and meta-analysis. Reproductive biomedicine online, 25(6), 561-571.

Pritts, E. A. (2001). Fibroids and infertility: a systematic review of the evidence. Obstetrical \& gynecological survey, 56(8), 483 .491-

Promberger, R., Simek, I.-M., Nouri, K., Obermaier, K., Kurz, C., \& Ott, J. (2018). Accuracy of tubal patency assessment in diagnostic hysteroscopy compared with laparoscopy in infertile women: a retrospective cohort study. Journal of Minimally Invasive Gynecology, 25(5), 794-799 .

Pundir, J., \& Toukhy, T. E. (2010). Uterine cavity assessment prior to IVF. Women's health, 6(6), 841-848.

Raju, G. R., Kumari, G. S., Krishna, K., Prakash, G., \& Madan, K. (2006). Assessment of uterine cavity by hysteroscopy in assisted reproduction programme and its influence on pregnancy outcome. Archives of gynecology and obstetrics, 274(3), 160-164 .

Shohayeb, A., \& El-Khayat, W. (2012). Does a single endometrial biopsy regimen (S-EBR) improve ICSI outcome in patients with repeated implantation failure? A randomised controlled trial. 
European Journal of Obstetrics \& Gynecology and Reproductive Biology, 164(2), 176-179.

Taylor, E., \& Gomel, V. (2008). The uterus and fertility. Fertility and sterility, 89(1), $1.16-$

Ugboaja, J., Oguejiofor, C., Igwegbe, A., \& Oranu, E. (2019). Abnormal hysteroscopy findings among a cross section of infertile Nigerian women. Nigerian Journal of Clinical Practice, 22)(1

Ugboaja, J. O., Oguejiofor, C. B., \& Ogelle, O. M. (2018. .Audit of operative hysteroscopies among infertile women in a resource - poor setting. International Journal of Gynecology \& Obstetrics, 141(1), 57-62 .

Yanaihara, A., Yorimitsu, T., Motoyama, H., Iwasaki, S., \& Kawamura, T. (2008). Location of endometrial polyp and pregnancy rate in infertility patients. Fertility and sterility, 90(1), 180-182 .

Yang, J.-H., Chen, M.-J., \& Yang, P.-K. (2019). Factors increasing the detection rate of intrauterine lesions on hysteroscopy in infertile women with sonographically normal uterine cavities. Journal of the Formosan Medical Association, 118(1), 488-493 . 
مدى انتثار ونمط نتائج تنظير الرحم غير الطبيعية بين مرضى العقم في مركز البيضاء للخصوية / ليبيا أغزيل سعد الحداد * 1 وزمزم شعبان 2

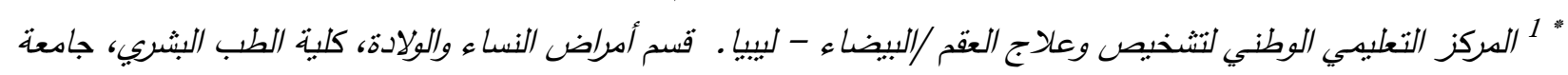
عمر الدختار ، البيضاء لبييا.

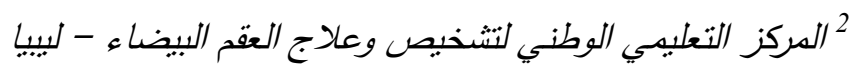

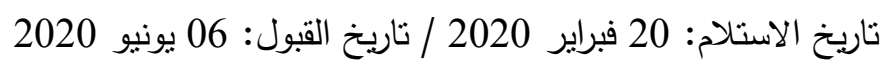
https://doi.org/10.54172/mjsc.v35i1.212 :Doi

المستخلص: دراسة مستقبلية تم إجرائها داخل المركز التعليمي الوطني لتتخيص وعلاج العقم/البيضاء - ليبيا وذلك لتقدير نسبة

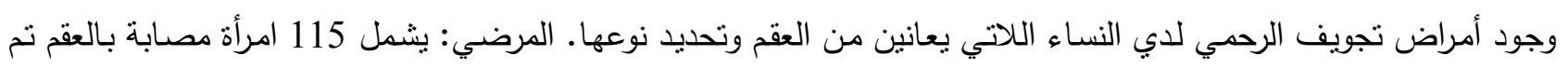

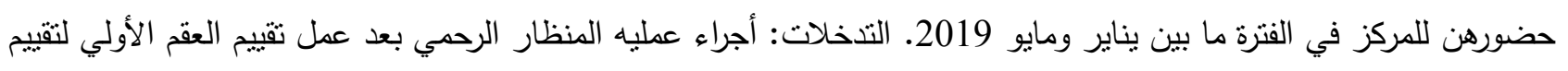

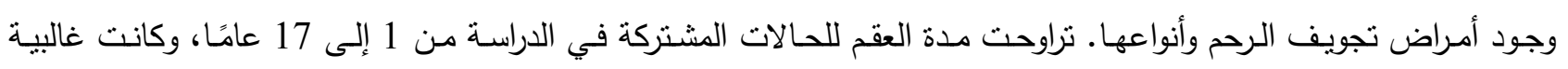

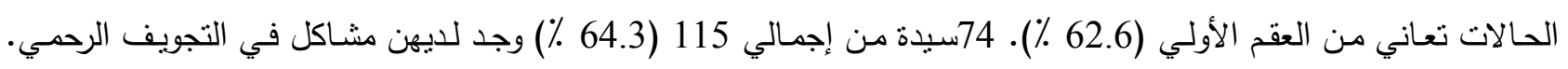

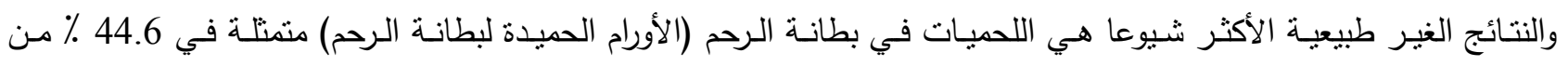

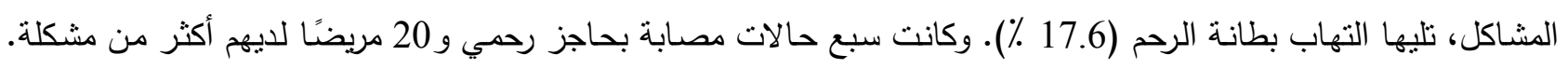

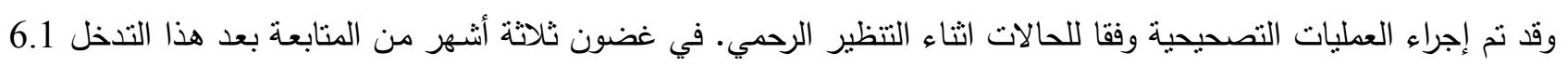

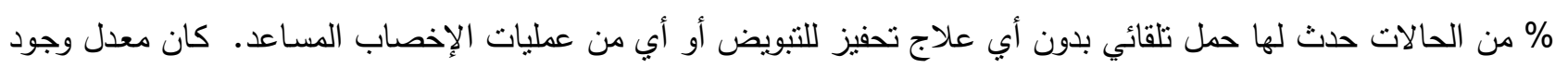

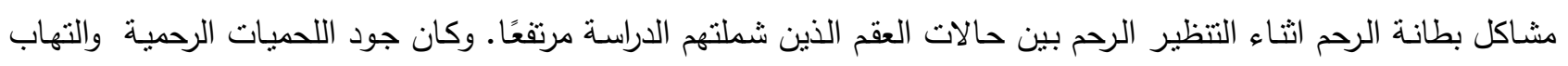

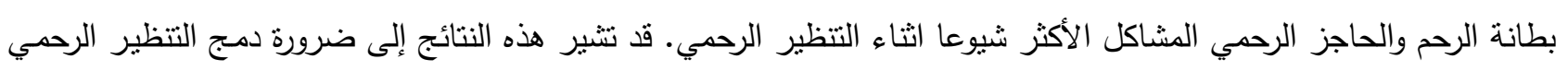
في النقييم الروتيني لحالات العقم.

الكلمات المفتاحية: المنظار الرحمي، العقم، مشاكل التجويف الرحمي.

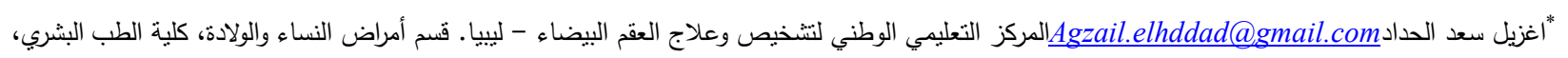

